Logic and Logical Philosophy

Volume 7 (1999), 101-115

\title{
Seiki Akama
}

\section{NELSON'S PARACONSISTENT LOGICS}

\begin{abstract}
David Nelson's constructive logics with strong negation may be viewed as alternative paraconsistent logic. These logics have been developed before da Costa's works. We address some philosophical aspects of Nelson's logics and give technical results concerning Kripke models and tableau calculi. We also suggest possible applications of paraconsistent constructive logics.
\end{abstract}

\section{Introduction}

da Costa [10] is one of the founding fathers of the so-called paraconsistent logics, which is a class of logical systems allowing inconsistency without triviality. Independently of da Costa's work, David Nelson [17] suggested a paraconsistent logic as a version of his constructive logics with strong negation. (But, Nelson seemed to aware of Jaśkowski's [14] work.) Nelson's work is of special interest in relation to the history of the development of paraconsistent logics. Unfortunately, Nelson's paraconsistent constructive logics have been neglected in the study of paraconsistent logics, and da Costa did not appear to be familiar with Nelson's systems. In this regard, Nelson's logics may be seen as alternative paraconsistent logics.

In this paper, we investigate Nelson's paraconsistent constructive logics from both a philosophical and a technical point of view. The main result presented in this paper is the tableau method of Nelson's systems. In section 2, we introduce Nelson's systems and their Kripke semantics. In section 3, we introduce a tableau formulation and provide a completeness result using Kripke models. In section 4, we discuss the philosophical significance and suggest possible applications of paraconsistent constructive logics to philosophy and computer science. 


\section{Constructive logics with strong negation}

Nelson [16] proposed an extension of positive intuitionistic logic with a new connective for constructible falsity or strong negation to overcome the nonconstructive features of intuitionistic negation. Independently, Markov [15] also developed a similar idea by showing that intuitionistic negation is definable by strong negation and intuitionistic implication. Constructive logics with strong negation have been extensively studied by logicians for many years. The paraconsistent constructive predicate logic with strong negation $N^{-}$of Almukdad and Nelson [9] is an extension of the positive intuitionistic logic with the following axioms for strong negation $(\sim)$ :

$$
\begin{aligned}
& \sim \sim A \leftrightarrow A, \\
& \sim(A \rightarrow B) \leftrightarrow(A \& \sim B), \\
& \sim(A \& B) \leftrightarrow(\sim A \vee \sim B), \\
& \sim(A \vee B) \leftrightarrow(\sim A \& \sim B), \\
& \sim \forall x A(x) \leftrightarrow \exists x \sim A(x), \\
& \sim \exists x A(x) \leftrightarrow \forall x \sim A(x) .
\end{aligned}
$$

If we add the following axiom (N7) to $N^{-}$, we obtain $N$ of Nelson [16]:

$$
A \& \sim A \rightarrow B
$$

In $N$, we can define intuitionistic negation $(\neg)$ as follows:

$$
\neg A \leftrightarrow(A \rightarrow \sim A) .
$$

Clearly, strong negation is stronger than intuitionistic negation, namely

$$
\sim A \rightarrow \neg A,
$$

but the converse does not hold. The reader is also referred to Akama [8] and Almukdad and Nelson [9] for sequential formulations of Nelson's logics.

A Kripke semantics for strong negation was developed by Thomason [23]; also see Gurevich [13] and Akama [3,5]. A strong negation model for $N^{-}$is of the form $\left(W, \leq, w_{0}, v a l, D\right)$, where $W$ is a set of worlds with the distinguished world $w_{0}$ such that for all $w \in W: w_{0} \leq w ; \leq$ is a reflexive and transitive relation on $W \times W$, val is a three-valued valuation assigning 1 (true) or 0 (false), -1 (undefined) to the atomic formula $p(t)$ at $w \in W$ with parameter 
$t \in D(w)$ satisfying:

$$
\begin{aligned}
& \text { if } \operatorname{val}(w, p(t))=1 \text { and } w \leq v \text { then } \operatorname{val}(v, p(t))=1, \\
& \text { if } \operatorname{val}(w, p(t))=0 \text { and } w \leq v \text { then } \operatorname{val}(v, p(t))=0,
\end{aligned}
$$

and $D$ is a domain function from $W$ to a set of variables such that if $w \leq v$ then $D(w) \subseteq D(v)$. Note that $V(w, A)=-1$ iff neither $V(w, A)=1$ nor $V(w, A)=0$. Next, we define the function $V(w, A)$ for any formula $A$.

$$
\begin{aligned}
V(w, p(t))=1 & \text { iff } \quad v a l(w, p(t))=1 \text { for any atomic } p(t) \text { with } t \in D(w), \\
V(w, p(t))=0 & \text { iff } \quad v a l(w, p(t))=0 \text { for any atomic } p(t) \text { with } t \in D(w), \\
V(w, A \& B)=1 & \text { iff } V(w, A)=1 \text { and } V(w, B)=1, \\
V(w, A \& B)=0 & \text { iff } V(w, A)=0 \text { or } V(w, B)=0, \\
V(w, A \vee B)=1 & \text { iff } V(w, A)=1 \text { or } V(w, B)=1, \\
V(w, A \vee B)=0 & \text { iff } V(w, A)=0 \text { and } V(w, B)=0, \\
V(w, A \rightarrow B)=1 & \text { iff } \forall v \in W(w \leq v \text { and } V(v, A)=1 \Rightarrow V(v, B)=1), \\
V(w, A \rightarrow B)=0 & \text { iff } V(w, A)=1 \text { and } V(w, B)=0, \\
V(w, \sim A)=1 & \text { iff } V(w, A)=0, \\
V(w, \sim A)=0 & \text { iff } V(w, A)=1, \\
V(w, \forall x A(x))=1 & \text { iff } \forall v \in W(w \leq v \Rightarrow V(v, A(t))=1 \text { for any } t \in D(v)), \\
V(w, \forall x A(x))=0 & \text { iff } V(w, A(t))=0 \text { for some } t \in D(w), \\
V(w, \exists x A(x))=1 & \text { iff } V(w, A(t))=1 \text { for some } t \in D(w), \\
V(w, \exists x A(x))=0 & \text { iff } \forall v \in W(w \leq v \Rightarrow V(v, A(t))=0 \text { for any } t \in D(v)), \\
V(w, A \& \sim A)=1 & \text { for some } w \text { and for some formula } A .
\end{aligned}
$$

We say that $A$ is true iff $V\left(w_{0}, A\right)=1$. $A$ is valid, written $\models_{N^{-}} A$, iff it is true in all strong negation models. Due to the last clause, the axiom (N7) is not valid. This is one of the basic features of paraconsistent logics. A strong negation model for $N$ does not have the last clause. Alternatively, we can use a four-valued valuation in a strong negation model for $N^{-}$. A completeness proof for $N$ may be found in Akama [3,5]. Thomason [23] proved that $N$ with the constant domain axiom $(C D): \forall x(A(x) \vee B) \rightarrow(\forall x A(x) \vee B)$, where $x$ is not free in $B$, has a Kripke semantics with constant domains.

\section{Tableau calculi}

Tableau calculi (or semantic tableaux) can be regarded as a variant of Gentzen systems; see Smullyan [22]. Tableau calculi are used as the proof method 
for both classical and non-classical logics (cf. Rautenberg [21]). The main advantage of the use of tableau calculi is that proofs in tableau calculi are easy to understand. In addition, it is possible to provide a comprehensive argument of completeness proof.

A tableau calculus for the propositional part of $N$ can be found in Rautenberg [21], and Akama [8] described a first-order tableau for $N$. We here present an alternative presentation for $N^{-}$. According to Smullyan [22], we use the notion of signed formula. If $X$ is a formula, then $T X$ and $F X$ are signed formulas. $T X$ reads " $X$ is provable" and $F X$ reads " $X$ is not provable", respectively. If $S$ is a set of signed formulas and $A$ is a signed formula, then we simply write $\{S, A\}$ for $S \cup\{A\}$. A tableau calculus $T N^{-}$consists of axioms and reduction rules. Let $p$ be an atomic formula and $A$ and $B$ be formulas.

\section{Tableau calculus $T N^{-}$for $N^{-}$}

\section{Axioms:}

$$
\begin{gathered}
\{T p, F p\} \\
\{T \sim p, F \sim p\}
\end{gathered}
$$

\section{Reduction Rules:}

$$
\begin{array}{ll}
\frac{S, T(A \& B)}{S, T A, T B}(T \&) & \frac{S, F(A \& B)}{S, F A ; S, F B}(F \&) \\
\frac{S, T(A \vee B)}{S, T A ; S, T B}(T \vee) & \frac{S, F(A \vee B)}{S, F A, F B}(F \vee) \\
\frac{S, T(A \rightarrow B)}{S, F A ; S, T B}(T \rightarrow) & \frac{S, F(A \rightarrow B)}{S_{T}, T A, F B}(F \rightarrow) \\
\frac{S, T(\sim(A \& B))}{S, T(\sim A) ; S, T(\sim B)}(T \sim \&) & \frac{S, F(\sim(A \& B))}{S, F(\sim A), F(\sim B)}(F \sim \&) \\
\frac{S, T(\sim(A \vee B))}{S, T(\sim A), T(\sim B)}(T \sim \vee) & \frac{S, F(\sim(A \vee B))}{S, F(\sim A) ; S, F(\sim B)}(F \sim \vee) \\
\frac{S, T(\sim(A \rightarrow B))}{S, T A, T(\sim B)}(T \sim \rightarrow) & \frac{S, F(\sim(A \rightarrow B))}{S, F A ; S, F(\sim B)}(F \sim \rightarrow) \\
\frac{S, T(\sim \sim A)}{S, T A}(T \sim \sim) & \frac{S, F(\sim \sim A)}{S, F A}(F \sim \sim)
\end{array}
$$




$$
\begin{array}{ll}
\frac{S, T(\forall x A(x))}{S, T(A(a))}(T \forall) & \frac{S, F(\forall x A(x))}{S_{T}, F(A(a))^{*}}(F \forall) \\
\frac{S, T(\exists x A(x))}{S, T(A(a))^{*}}(T \exists) & \frac{S, F(\exists x A(x))}{S, F(A(a))}(F \exists) \\
\frac{S, T(\sim \forall x A(x))}{S, T(\sim A(a))^{*}}(T \sim \forall) & \frac{S, F(\sim \forall x A(x))}{S, F(\sim A(a))}(F \sim \forall) \\
\frac{S, T(\sim \exists x A(x))}{S, T(\sim A(a))}(T \sim \exists) & \frac{S, F(\sim \exists x A(x))}{S_{T}, F(\sim A(a))^{*}}(F \sim \exists)
\end{array}
$$

Here, ${ }^{*}$ denotes the variable restriction that the parameter $a$ introduced must not occur in any formula of $S$ or in the formula $A(x)$. $S_{T}$ stands for $\{T X \mid T X \in S\}$. A proof of a sentence $X$ is a closed tableau for $F X$. A tableau is a tree constructed by the above reduction rules. A tableau is closed if each branch is closed. A branch is closed if it contains the axioms of the form (AX1) or (AX2). We write $\vdash_{T N^{-}} A$ to mean that $A$ is provable in $T N^{-}$. A tableau calculus $T N$ for $N$ needs an additional axiom: (AX3) $\{T p, T \sim p\}$.

Now, we prove the completeness of the tableau calculus $T N^{-}$with respect to strong negation models. Although we know that $N^{-}$is complete, it would be interesting to show that $T N^{-}$is a complete proof proceduce. Our strategy is similar to that for the tableau for intuitionistic logic sketched in Fitting [12]. It is slightly different from the one presented in Akama [8].

Let $S=\left\{T X_{1}, \ldots, T X_{n}, F Y_{1}, \ldots, F Y_{m}\right\}$ be a set of signed formula, $\left(W, \leq, w_{0}, v a l, D\right)$ be a strong negation model, and $w \in W$. We say that $w$ refutes $S$ if

$$
\begin{aligned}
& V\left(w, X_{i}\right)=1 \text { if } \quad T X_{i} \in S, \\
& V\left(w, X_{i}\right) \neq 1 \text { if } F X_{i} \in S .
\end{aligned}
$$

A set $S$ is refutable if something refutes it. If $S$ is not refutable, it is valid.

Theorem 1 (Soundness of $T N^{-}$). If $A$ is provable, then $A$ is valid.

Proof. If $A$ is of the form of axioms, it is easy to see that it is valid. For reduction rules, it suffices to check that they preserve validity. For example. consider the rule $(T \sim \vee)$. We have to show that if $S, T(\sim(A \vee B))$ is refutable then $S, T(\sim A), T(\sim B)$ is also refutable. By the assumption, there is a strong negation model $\left(W, \leq, w_{0}, v a l, D\right)$, in which $w_{0}$ refutes $S$ and $V\left(w_{0}, \sim(A \vee\right.$ 
$B)=1$. This implies:

$$
\begin{array}{lll}
V\left(w_{0}, A \vee B\right)=0 & \text { iff } & V\left(w_{0}, A\right)=0 \text { and } V\left(w_{0}, B\right)=0 \\
& \text { iff } \quad V\left(w_{0}, \sim A\right)=1 \text { and } V\left(w_{0}, \sim B\right)=1 .
\end{array}
$$

Therefore, $S, T(\sim A), T(\sim B)$ is shown to be refutable.

Next, consider the rule $(F \sim \rightarrow)$. By the assumption, there is a strong negation model $\left(W, \leq, w_{0}, v a l, D\right)$, in which $w_{0}$ refutes $S$ and $V\left(w_{0}, \sim(A \rightarrow\right.$ $B)) \neq 1$. This implies:

$$
V\left(w_{0}, A \rightarrow B\right) \neq 0 \text { iff } V\left(w_{0}, A\right) \neq 1 \text { and } V\left(w_{0}, \sim B\right) \neq 1 .
$$

Therefore, $S, F A$ and $S, F(\sim B)$ are refutable.

Next, consider the rule $(T \sim \forall)$. By the assumption, we have a strong negation model $\left(W, \leq, w_{0}, v a l, D\right)$, in which $w_{0}$ refutes $S$ and $V\left(w_{0}\right.$, $\sim \forall x A(x))=1$. This implies:

$$
\begin{array}{lll}
V\left(w_{0}, \sim \forall x A(x)\right)=1 \quad \text { iff } & V\left(w_{0}, \forall x A(x)\right)=0 \\
& \text { iff } & V\left(w_{0}, A(a)\right)=0 \text { for some } a \in D\left(w_{0}\right) \\
& \text { iff } & V\left(w_{0}, \sim A(a)\right)=1 \text { for some } a \in D\left(w_{0}\right) .
\end{array}
$$

Here, $a$ is subject to the variable restriction. Then, $S, T(\sim A(a))$ is also refutable. We can similarly handle other cases.

We are now in a position to prove completeness of $T N^{-}$, which is the converse of theorem 1. The proof below is similar to the Henkin proof described in Akama $[3,5]$.

A finite set of signed formulas $\Gamma$ is non-trivial if no tableau for it is closed. An infinite set of signed formulas is non-trivial if every finite subset is nontrivial. If a set of formulas is not non-trivial, it is trivial. Every formula is provable from a trivial set.

Definition 1. Let $\boldsymbol{P}$ be a set of parameters and $\Gamma$ a set of signed formulas. We say that $\Gamma$ is maximally non-trivial with respect to $\boldsymbol{P}$ if

(1) every signed formula in $\Gamma$ uses only parameters of $\boldsymbol{P}$,

(2) $\Gamma$ is non-trivial,

(3) for every formula $A$ with parameters in $\boldsymbol{P}$, either $T A \in \Gamma$ or $F A \in \Gamma$.

We write $L(C)$ to denote the new language extending the set of constants $C_{L}$ of the original language $L$ with a set of constants $C=\left\{c_{1}, \ldots, c_{n}\right\}$. 
Definition 2. We say that a non-trivial set of signed formulas $\Gamma$ is $C$ saturated if

(1) $\Gamma$ is maximally non-trivial with respect to $C_{L} \cup C$,

(2) if $T(\exists x A(x)) \in \Gamma$, then $T(A(c)) \in \Gamma$ for some $c \in C_{L} \cup C$,

(3) if $T(\sim \forall x A(x)) \in \Gamma$, then $T(\sim A(c)) \in \Gamma$ for some $c \in C_{L} \cup C$.

Lemma 1. A non-trivial set of signed formulas $\Gamma_{0}$ can be extended to a maximally non-trivial set of signed formulas $\Gamma$.

Proof. Since the language $L$ has a countably infinite set of sentences, we can enumerate sentences $A_{1}, A_{2}, \ldots$ Now, we define for a non-trivial set of signed formulas $\Gamma_{0}$ a sequence of non-trivial sets of signed formulas $\Gamma_{0}, \Gamma_{1}$, $\Gamma_{2}, \ldots$ in the following way:

$$
\Gamma_{n+1}= \begin{cases}\Gamma_{n} \cup\left\{T A_{n+1}\right\} & \text { if } \Gamma_{n} \cup\left\{T A_{n+1}\right\} \text { is non-trivial, } \\ \Gamma_{n} \cup\left\{F A_{n+1}\right\} & \text { if } \Gamma_{n} \cup\left\{F A_{n+1}\right\} \text { is non-trivial, } \\ \Gamma_{n} & \text { otherwise. }\end{cases}
$$

Then, we set

$$
\Gamma=\bigcup \Gamma_{i}
$$

It is obvious that $\Gamma$ satisfies the desired properties of a maximally non-trivial set.

Lemma 2. A non-trivial set $\Gamma$ of signed formulas in $L$ can be extended to a $C$-saturated non-trivial set $\Delta$ of signed formulas in $L(C)$.

Proof. Let $\Gamma_{0}=\Gamma$. Extend $\Gamma_{0}$ to a set $\Gamma_{1}$ maximally non-trivial with respect to $C_{L}$. Since $C=\left\{c_{1}, c_{2}, \ldots\right\}$ is a countable set of constants not in $L$. we can enumerate sentences of the form $\exists x A(x)$ in $L(C)$ as $\exists x A_{1}(x)$, $\exists x A_{2}(x), \ldots$ By definition $\forall x A(x) \leftrightarrow \sim \exists x \sim A(x)$, we here only consider the case of $\exists x A(x) . \Gamma_{i}$ can be then defined for any $n \geq 1$ as follows:

$(i=2 n)$ Take the first formula of the form $\exists x A_{m}(x)$. If $T(\exists x A(x)) \in$ $\Gamma_{n-1}$ but $T(A(c)) \notin \Gamma_{n-1}$ for all $c \in C_{L} \cup\left\{c_{1}, \ldots, c_{n}\right\}$, then set $\Gamma_{2 n}=$ $\Gamma_{2 n-1} \cup\left\{T\left(A\left(c_{1}\right)\right)\right\}$. We need a similar construction on the formula of the form $\sim \forall x A_{m}(x)$.

$(i=2 n+1)$ By Lemma 1 , we extend $\Gamma_{2 n}$ to $\Gamma_{2 n+1}$, which is maximally non-trivial with respect to $C_{L} \cup C$.

Then, we define $\Delta=\bigcup \Gamma_{i}$. It suffices to check that $\Delta$ is $C$-saturated. Because each $\Gamma_{n}$ is non-trivial, $\Delta$ is also non-trivial. Let $A(c)$ be any sentence 
of $L(C)$ with $c \in C$. Due to the maximality of $\Gamma_{2 n+1}$, one of the conditions $T A \in \Gamma_{2 n+1}, F A \in \Gamma_{2 n+1}$, or $\Gamma_{2 n+1} \cup\{T A\}$ and $\Gamma_{2 n+1} \cup\{F A\}$ are provable, holds. Thus, $\Delta$ is shown to be maximally in $L(C)$. Finally, $T(\exists x A(x)) \in \Delta$ (also $T(\sim \forall x A(x) \in \Delta)$. We suppose $\exists x A_{n}(x)$ (also $\sim \forall x A(x)$ ), i.e. the $n$-th enumeration. From the above construction, $T(A(c)) \in \Delta$ (also $T(\sim A(c) \in$ $\Delta$ ) for some $c \in C_{L} \cup\left\{c_{1}, \ldots, c_{n}\right\}$ must be satisfied. This implies that $\Delta$ is $C$-saturated.

We here define a canonical strong negation model with respect to the tableau:

Definition 3. Let $C_{0}, C_{1}, C_{2}, \ldots$ be a countable sequence of disjoint countable sets of constants not occurring in $L$. We denote $C_{0} \cup C_{1} \cup \ldots \cup C_{n}$ by $C_{n}^{*}$. Then, we define a canonical strong negation model $\left(W, \leq, w_{0}, v a l, D\right)$ as follows:

(1) $W=\left\{\Gamma \mid \Gamma\right.$ is $C_{n}^{*}$-saturated in $L\left(C_{n}^{*}\right)$ for some $\left.n\right\}$.

(2) If $\Gamma$ is $C_{n}^{*}$-saturated and $L(\Gamma)=L \cup C_{n}^{*}$, then $D(\Gamma)=C_{n}^{*}$.

(3) Define $\leq$ in the following way:

$$
\Gamma \leq \Delta \text { iff } T A \in \Gamma \Rightarrow T A \in \Delta \text { and } D(\Gamma) \subseteq D(\Delta) .
$$

(4) For any $\Gamma \in W$ and atomic formula $p(c)$ with $c \in D(\Gamma)$, we define the three-valued valuation $v a l$ as:

$$
\operatorname{val}(\Gamma, p(c))= \begin{cases}1 & \text { if } T(p(c)) \in \Gamma \\ 0 & \text { if } T(\sim p(c)) \in \Gamma \\ -1 & \text { otherwise }\end{cases}
$$

We can easily verify that a canonical strong negation model satisfies the desired properties of a strong negation model.

Lemma 3. For any $\Gamma \in W$ in a canonical strong negation model $(W, \leq$, $v_{0}$, val, $\left.D\right)$, we have the following properties:

(1) if $T(A \& B) \in \Gamma$, then $T A \in \Gamma$ and $T B \in \Gamma$,

(2) if $F(A \& B) \in \Gamma$, then $F A \in \Gamma$ or $F B \in \Gamma$,

(3) if $T(A \vee B) \in \Gamma$, then $T A \in \Gamma$ or $T B \in \Gamma$,

(4) if $F(A \vee B) \in \Gamma$, then $F A \in \Gamma$ and $F B \in \Gamma$,

(5) if $T(A \rightarrow B) \in \Gamma$, then $F A \in \Gamma$ or $T A \in \Gamma$,

(6) if $F(A \rightarrow B) \in \Gamma$, then for some $\Delta$ such that $\Gamma \leq \Delta, T A \in \Delta$ and $F B \in \Delta$, 
(7) $\quad$ if $T(\sim(A \& B)) \in \Gamma$, then $T(\sim A) \in \Gamma$ or $T(\sim B) \in \Gamma$,

(8) if $F(\sim(A \& B)) \in \Gamma$, then $F(\sim A) \in \Gamma$ and $F(\sim B) \in \Gamma$,

(9) if $T(\sim(A \vee B)) \in \Gamma$, then $T(\sim A) \in \Gamma$ and $T(\sim B) \in \Gamma$,

(10) if $F(\sim(A \vee B)) \in \Gamma$, then $F(\sim A) \in \Gamma$ or $F(\sim B) \in \Gamma$,

(11) if $T \sim(A \rightarrow B) \in \Gamma$, then $T A \in \Gamma$ and $T(\sim B) \in \Gamma$,

(12) if $F \sim(A \rightarrow B) \in \Gamma$, then $F A \in \Gamma$ or $F(\sim B) \in \Gamma$,

(13) if $T(\sim \sim A) \in \Gamma$, then $T A \in \Gamma$,

(14) if $F(\sim \sim A) \in \Gamma$, then $F A \in \Gamma$,

(15) if $T(\forall x A(x)) \in \Gamma$, then $T(A(a)) \in \Gamma$ for all $a \in D(\Gamma)$,

(16) if $F(\forall x A(x)) \in \Gamma$, then for some $\Delta$ such that $\Gamma \leq \Delta, F(A(a)) \in \Delta$ for some $a \in D(\Delta)$,

(17) if $T(\exists x A(x)) \in \Gamma$, then $T(A(a)) \in \Gamma$ for some $a \in D(\Gamma)$,

(18) if $F(\exists x A(x)) \in \Gamma$, then $F(A(a)) \in \Gamma$ for all $a \in D(\Gamma)$,

(19) if $T(\sim \forall x A(x)) \in \Gamma$, then $T(\sim A(a)) \in \Gamma$ for some $a \in D(\Gamma)$,

(20) if $F(\sim \forall x A(x)) \in \Gamma$, then $F(\sim A(a)) \in \Gamma$ for all $a \in D(\Gamma)$,

(21) $\quad$ if $T(\sim \exists x A(x)) \in \Gamma$, then $T(\sim A(a)) \in \Gamma$ for all $a \in D(\Gamma)$,

(22) if $F(\sim \exists x A(x)) \in \Gamma$, then for some $\Delta$ such that $\Gamma \leq \Delta, F(\sim A(a)) \in \Delta$ for some $a \in D(\Delta)$.

Proof. For (1), assume that $T(A \& B) \in \Gamma$ but $T A \notin \Gamma$. If $\Gamma, T(A \& B)$ is non-trivial, then $\Gamma, T(A \& B), T A$ is also non-trivial. But, it contradicts the maximality of $\Gamma$. Thus, $T A \in \Gamma$. Similarly, $T B \in \Gamma$. Similar reasoning can be applied to (4), (8), (9), (11), (13) and (14).

For (3), assume that $T A \notin \Gamma$ and $T B \notin \Gamma$. Since $\Gamma$ is maximal, both $\Gamma, T A$ and $\Gamma, T B$ are inconsistent (i.e. provable). For a finite subset $S$ of $\Gamma$, both $S, T A$ and $S, T B$ are inconsistent. By $(T \vee), S, T(A \vee B)$ is also provable. This implies that $\Gamma, T(A \vee B)$ is provable and $T(A \vee B) \notin \Gamma$. We can justify (2), (5), (7), (10) and (12) in the similar way.

For (6), by $F(A \rightarrow B) \in \Gamma$ we have $\Gamma_{T}, T A, F B$ is not provable. By Lemma $1, \Gamma_{T}, T A, F B$ can be extended to a maximally non-trivial set $\Delta$ such that $T A \in \Delta$ and $F B \in \Delta$.

For (15), assume $T(\forall x A(x)) \in \Gamma$ but $T(A(a)) \notin \Gamma$ for all $a \in D(\Gamma)$. If $\Gamma, T(\forall x A(x))$ is consistent, so is $\Gamma, T(\forall x A(x)), T(A(a))$. So $\Gamma$ is not maximal. Thus, $T(A(a)) \in \Gamma$. We can similarly deal with (17), (19) and (21). 
For (16), assume $F(\forall x A(x)) \in \Gamma$. If $a$ does not occur in $\Gamma$, then $\Gamma_{T}, F(A(a))$ is consistent. By lemma 2 , we can extend $\Gamma_{T}, F(A(a))$ to $\Delta$ such that $F(A(a)) \in \Delta$ for some $a \in D(\Delta)$. Similarly, for (18), (20) and (22).

Theorem 2. For any $\Gamma \in W$ in a canonical strong negation model and any formula $A$,

$$
\begin{aligned}
& T A \in \Gamma \quad \text { iff } \quad V(\Gamma, A)=1, \\
& F A \in \Gamma \quad \text { iff } \quad V(\Gamma, A) \neq 1 .
\end{aligned}
$$

Proof. By induction on $A$. The case $A$ is an atomic formula is immediate.

(1) $A=B \& C($ also $\sim(B \vee C))$ :

$$
\begin{array}{rll}
T(B \& C) \in \Gamma \quad \text { iff } & T B \in \Gamma \text { and } T C \in \Gamma \\
& \text { iff } & V(\Gamma, B)=1 \text { and } V(\Gamma, C)=1 \\
\text { iff } & V(\Gamma, B \& C)=1 . \\
F(B \& C) \in \Gamma \quad \text { iff } & F B \in \Gamma \text { or } F C \in \Gamma \\
\text { iff } & V(\Gamma, B) \neq 1 \text { or } V(\Gamma, C) \neq 1 \\
\text { iff } & V(\Gamma, B \& C) \neq 1 .
\end{array}
$$

(2) $A=B \vee C($ also $\sim(B \& C))$ :

$$
\begin{array}{rll}
T(B \vee C) \in \Gamma \quad \text { iff } & T B \in \Gamma \text { or } T C \in \Gamma \\
\text { iff } & V(\Gamma, B)=1 \text { or } V(\Gamma, C)=1 \\
\text { iff } & V(\Gamma, B \vee C)=1 . \\
F(B \vee C) \in \Gamma \quad \text { iff } & F B \in \Gamma \text { and } F C \in \Gamma \\
\text { iff } & V(\Gamma, B) \neq 1 \text { and } V(\Gamma, C) \neq 1 \\
\text { iff } & V(\Gamma, B \vee C) \neq 1 .
\end{array}
$$

(3) $A=B \rightarrow C$ :

$$
\begin{aligned}
& T(B \rightarrow C) \in \Gamma \quad \text { iff } \quad \forall \Delta \geq \Gamma(T(B \rightarrow C) \in \Delta) \\
& \text { iff } \forall \Delta \geq \Gamma(F B \in \Delta \text { or } T C \in \Delta) \\
& \text { iff } \forall \Delta \geq \Gamma(V(\Delta, B) \neq 1 \text { or } V(\Delta, C)=1) \\
& \text { iff } V(\Gamma, B \rightarrow C)=1 \text {. } \\
& F(B \rightarrow C) \in \Gamma \quad \text { iff } \quad \exists \Delta \geq \Gamma(T B \in \Delta \text { and } T C \in \Delta) \\
& \text { iff } \exists \Delta \geq \Gamma(V(\Delta, B)=1 \text { and } V(\Delta, C) \neq 1) \\
& \text { iff } V(\Gamma, B \rightarrow C) \neq 1 \text {. }
\end{aligned}
$$


(4) $A=\sim \sim B$

$$
\begin{array}{rll}
T(\sim \sim B) \in \Gamma \quad \text { iff } & T B \in \Gamma \\
\text { iff } & V(\Gamma, B)=1 \\
\text { iff } & V(\Gamma, \sim B)=0 \\
\text { iff } & V(\Gamma, \sim \sim B)=1 . \\
F(\sim \sim B) \in \Gamma & \text { iff } & F B \in \Gamma \\
\text { iff } & V(\Gamma, B) \neq 1 \\
\text { iff } & V(\Gamma, \sim B) \neq 0 \\
\text { iff } & V(\Gamma, \sim \sim B) \neq 1 .
\end{array}
$$

(5) $A=\exists x B(x)$ (also $\sim \forall x A(x)$ ):

$$
\begin{array}{lll}
T(\exists x B(x)) \in \Gamma \quad \text { iff } & T(B(a)) \in \Gamma \text { for some } a \in D(\Gamma) \\
& \text { iff } & V(\Gamma, B(a))=1 \text { for some } a \in D(\Gamma) \\
\text { iff } & V(\Gamma, \exists x B(x))=1 . \\
F(\exists x B(x)) \in \Gamma \quad \text { iff } & F(B(a)) \in \Gamma \text { for all } a \in D(\Gamma) \\
\text { iff } & V(\Gamma, B(a)) \neq 1 \text { for all } a \in D(\Gamma) \\
\text { iff } & V(\Gamma, \exists x B(x)) \neq 1 .
\end{array}
$$

(6) $A=\forall x B(x)$ (also $\sim \exists x B(x)$ ):

$$
\begin{aligned}
& T(\forall x B(x)) \in \Gamma \quad \text { iff } \forall \Delta \geq \Gamma(T(B(a)) \in \Delta \text { for all } a \in D(\Delta)) \\
& \text { iff } \quad \forall \Delta \geq \Gamma(V(\Delta, B(a))=1 \text { for all } a \in D(\Delta)) . \\
& \text { iff } \quad V(\Gamma, \forall x B(x))=1 \\
& F(\forall x B(x)) \in \Gamma \quad \text { iff } \exists \Delta \geq \Gamma(F(B(a)) \in \Delta \text { for some } a \in D(\Delta) \\
& \text { iff } \exists \Delta \geq \Gamma(V(\Delta, B(a)) \neq 1 \text { for some } a \in D(\Delta)) \\
& \text { iff } V(\Gamma, \forall x B(x)) \neq 1 . \quad \square
\end{aligned}
$$

As a consequence, we obtain the completeness of $T N$ :

Theorem 3 (Completeness Theorem). $\vdash_{T N^{-}} A$ iff $\models_{N^{-}} A$.

Proof. The soundness was already proved in theorem 1. For the completeness, it suffices to show that an open tableau is refutable by a counter strong negation model by theorem 2. By contraposition, we reach the completeness theorem. 
We can also see that $N^{-}$is a paraconsistent logic.

Theorem 4. $A \& \sim A \rightarrow B$ is not provable in $N^{-}$.

Proof. Due to the lack of (AX3) in $T N^{-}$, we cannot prove the formula. This means that in $N^{-}$both $A$ and $\sim A$ can be true at some world $w$ for some formula $A$ as in other paraconsistent logics.

\section{Discussion}

We have addressed the logical aspects of Alumkdad and Nelson's paraconsistent constructive logic $N^{-}$. The Nelson systems strengthen the concept of negation in intuitionistic logic in that strong negation is constructible. In addition, strong negation has rather surprising features concerning a contradiction. Namely, all contradictions are equivalent in both classical and intuitionistic logic, but they may not be equivalent in $N^{-}$. We also observe that $A \leftrightarrow \sim A$ can be consistently added as an axiom for some formula $A$ in $N^{-}$. Like all paraconsistent logics, $N^{-}$can form an inconsistent, but non-trivial, theory.

Now, we compare $N^{-}$with da Costa's projects on paraconsistent logics initiated in 1974. First, da Costa used classical logic as the strating point, whereas Nelson used intuitionistic logic as the basis. Secondly, the da Costa constructions of non-triviasl theories in $C_{\omega}$ or related systems rely on pedagogical ideas to torelate contradictions. Thirdly, the semantics for the da Costa systems are not always intuitive. The Kripke semantics for $\mathrm{N}^{-}$ has the intuitive appeal. In fact, strong negation can be interpreted as the refutability. On these grounds, Nelson should be counted as a forerunner of paraconsistent logics.

Why did not Nelson explicitly develop "paraconsistent" logics? Indeed Nelson did formulate a paraconsistent logic in Nelson [17], but some discussions may be in order. For instance, we can find the following remarks (p. 224); "The system has been constructed, of course, to show that the logical operations may be interpreted in such a way that a mathematical system for arithmetic may be inconsistent without being overcomplete." A similar observation also appeared in Almukdad and Nelson [9]. The constructive system $S$ in Nelson [17] is slightly different from $N^{-}$. Technically, $S$ is a variant of $N^{-}$by deleting contraction and adding contraposition. Similar systems were also investigated by Zaslavskii [28] and Akama [6, 7]. These works reveal that paraconsistency is closely related to structural rules in the sense of Genzen systems (cf. Wansing [26]). In this regard, further work is 
needed to address the connection of subsystems of $N^{-}$and substructural logics. We should also point out that the da Costa constructions using the formula of the form $A^{\circ}$ can also be made in the Nelson systems. But, Nelson's logics can dispense with da Costa's ideas to construct a paraconsistent system.

Nelson [17] continued, "Does the system have any practical interest? I should not want to claim much in this direction." For mathematicians, Nelson's logics are alternative to Heyting's intuitionistic logic. It is thus clear that paraconsistent mathematics is possible in the arithmetic based on $N^{-}$. Philosophers appear to be interested in paraconsistent constructive logics. For example, Fitch [11] proposed a similar system with strong negation to overcome paradoxes. Prawitz [20] firstly noted the usefultness of $N^{-}$ for naive set theory in the context of natural deduction. The line of investigation was recently explored by Akama [4, 7]. The contractionless fragment of $N^{-}$is capable of giving a formalization of naive set theory. The paraconsistent constructive logics are also attracted to those who are working on computer science, in particular, artificial intelligence. Pearce and his students showed that Nelson's logics can serve as the foundation for logic programming in Pearce and Wagner [18, 19] and Wagner [24, 25]; also see Akama [6]. Wagner [25] and Wansing [27] also tried to employ strong negation systems for non-monotonic reasoning. Akama [8] recently sketched another tableau formulation of $\mathrm{N}^{-}$, providing a wider fragment of logic programming with strong negation. We believe that Nelson's paraconsistent constructive logics deserve further investigations from both foundational and practical point of view.

Acknowledgments. We are grateful to a referee for constructive comments.

\section{References}

[1] Akama, S. (1987): Resolution in constructivism, Logique et Analyse 120, 385399.

[2] Akama, S. (1988a): On the proof method for constructive falsity, Zeitschrift für mathematische Logik und Grundlagen der Mathematik 34, 385-392.

[3] Akama, S. (1988b): Constructive predicate logic with strong negation and model theory, Notre Dame Journal of Formal Logic 29, 18-27.

[4] Akama, S. (1989): Constructive Falsity: Foundations and Their Applications to Computer Science, Ph.D. dissertation, Department of Administration Engineering, Keio University, Yokohama, Japan. 
[5] Akama, S. (1990): Subformula semantics for strong negation systems, The Journal of Philosophical Logic 19, 217-226.

[6] Akama, S. (1995): Three-valued constructive logic and logic programs, Proc. of the IEEE 25th International Symposium on Multiple-Valued Logic, 276-281, Bloomington, USA, May.

[7] Akama, S. (1996): Curry's paradox in contractionless constructive logic, The Journal of Philosophical Logic 25, 217-226.

[8] Akama, S. (1997): Tableaux for logic programming with strong negation, D. Galmiche (ed.), TABLEAUX'9\%: Automated Reasoning with Analytic Tableaux and Related Methods, 31-42, Springer, Berlin.

[9] Almukdad, A., and D. Nelson (1984): Constructible falsity and inexact predicates, The Journal of Symbolic Logic 49, 8-37, 231-233.

[10] da Costa, N. C. A.: (1974): On the theory of inconsistent formal system, Notre Dame Journal of Formal Logic 15, 497-510.

[11] Fitch, F. (1952): Symbolic Logic, Ronald Press, New York.

[12] Fitting, M. (1969): Intuitionistic Logic, Model Theory and Forcing, NorthHolland, Amsterdam.

[13] Gurevich, Y. (1977): Intuitionistic logic with strong negation, Studia Logica $36,49-59$.

[14] Jaśkowski, S. (1948): Un calcul des propositions pour les systems déductifs contradictories, Studia Sicietatis Scientiarum Torunensis, Sect. A, 1, 57-77.

[15] Markov, A. A. (1950): Constructive logic (in Russian), Uspekhi Mathematičekih Nauk 5, 187-188.

[16] Nelson, D. (1949): Constructible falsity, The Journal of Symbolic Logic 14, $16-26$.

[17] Nelson, D. (1959): Negation and separation of concepts in constructive systems, A. Heyting (ed.), Constructivity in Mathematics, 208-225, NorthHolland, Amsterdam.

[18] Pearce, D., and G. Wagner (1990): Reasoning with negative information I: Strong negation in logic programs, Acta Philosophica Fennica 49, 430-453.

[19] Pearce, D., and G. Wagner (1991): Logic programming with strong negation, P. Schroeder-Heister (ed.), Extensions in Logic Programming, 311-326, Springer, Berlin.

[20] Prawitz, D. (1965): Natural Deduction: A Proof-Theoretical Study, Almqvist and Wiksell, Stockholm. 
[21] Rautenberg, W. (1979): Klassische und Nichtklassische Aussagenlogik, Vieweg, Wiesbaden.

[22] Smullyan, R. (1968): First-Order Logic, Springer, Berlin.

[23] Thomason, R. H. (1969): A semantical study of constructible falsity, Zeitschrift für mathematische Logik und Grundlagen der Mathematik 15, 247-257.

[24] Wagner, G. (1991): Logic programming with strong negation and inexact predicates, Journal of Logic and Computation 1, 835-859.

[25] Wagner, G. (1992): Vivid Logic: Knoweldge-Based Reasoning with Two Kinds of Negation, Ph.D. dissertation, Freien Universität Berlin, also published as Lecture Notes in Aritificial Intelligence 764, Springer, Berlin, 1994.

[26] Wansing, H. (1992): The Logic of Information Structures, Ph.D. dissertation, Freien Universität Berlin, also published as Lecture Notes in Aritificial Intelligence 681, Springer, Berlin, 1993.

[27] Wansing, H. (1995): Semantics-based nonmonotonic inference, Notre Dame Journal of Formal Logic 36, 44-54.

[28] Zaslavskii, I. (1978): Symmetric Constructive Logics (in Russian), Erevan.

\section{Seiki Akama}

Computational Logic Laboratory

Department of Information Systems

Teikyo Heisei University

2289 Uruido, Ichihara-shi

Chiba, 290-0193, Japan

akama@thu.ac.jp 